Chapter 10

Assembly System Integration 


\title{
MAN-ROBOT COOPERATION - NEW TECHNOLOGIES AND NEW SOLUTIONS
}

\author{
Timo Salmi*, Ilari Marstio*, Timo Malmº, Esa Laine ${ }^{\circ}$ \\ *VTT, P.O. Box 1000 \\ ${ }^{\circ}$ VTT, P.O. Box 1300 \\ 'TUT, P.O. Box 589 \\ FI-02044 VTT \\ FI-33101 Tampere \\ FI-33101 Tampere \\ Timo.Salmi@vtt.fi \\ Timo.Malm@vtt.fi \\ Esa.P.Laine@tut.fi
}

Ilari.Marstio@vtt.fi

\begin{abstract}
In this paper we present technologies that enable man-robot cooperation. Several robots are presented that are capable of working safely around humans. Software and sensor-based safety systems are also discussed. We review the regulations and robot standards which determine the construction of today's robot solutions. Finally, we present a few concept man-robot solutions in assembly cells from a safety perspective.
\end{abstract}

\section{Introduction}

The first industrial robots in the 1960s and 1970s were developed for tasks that were dangerous or very monotonous to man. Since then, robots have improved and brought flexibility to industrial automation. Compared to traditional rigid automation robots are reprogrammable and adaptable, and compared to man they are more precise and faster.

Nowadays, man and robot are completely isolated from each other for safety reasons. It must be ensured that the robot cannot hit anybody, and that nobody can access the robot without stopping it. Usually this is done by fencing in the robot, and often the enclosure is larger than the robot's workspace to prevent crushing accidents. This requires a lot of space.

Small patch sizes, assembly-to-order ideologies, and short life cycles of products are making large investments in rigid automation unprofitable. Often, manual assembly seems to be the solution to achieving flexibility. At the same time the component sizes and tolerances are getting smaller, causing many assembly tasks to be very challenging or even impossible to man.

In this present situation, combining the robot's accuracy and man's flexibility may be the solution to efficient production. When a worker needs to work very close to a robot it is not necessarily flexible, because the robot has to be stopped before anyone can enter its work area. In this paper we have surveyed different technologies and concept solutions to make man-robot cooperation more flexible,

Please use the following format when citing this chapter:

Salmi, T., Marstio, I., Malm, T., Laine, E., 2008, in IFIP International Federation for Information Processing, Volume 260, Micro-Assembly Technologies and Applications, eds. Ratchev, S., Koelemeijer, S., (Boston: Springer), pp. 385-394. 
mostly from a safety point of view. We do not address what the tasks are that humans and robots do, and the psychological aspect is omitted. [1]

\section{Technologies}

Safety is a core factor when bringing humans close to a robot. The working environment needs to be safe, yet the work should be flexible and effective. Many new technologies enable this. There are several ways of approaching the problem. Either the robot has to be made such that it cannot carry destructive force, or it has to be controlled by an intelligent controller and/or with safety sensors.

\subsection{Robots}

In recent years robot technology has undergone immense changes. Control and the user interface have become increasingly PC-based, and calculating power has multiplied. Robot structures have become more monolithic due to higher payload and accuracy demands. At the same time, ways of using robots have changed and therefore safety issues have become more important. Human-robot cooperation and research into it have promoted greater interest in safer robot structures and controlling technologies.

One approach to a safer small robot is the Neuronics Katana robot. The robot itself weighs only 4.3 kilograms and has a payload of 500 grams. It is shaped in such a way that sharp edges and the danger of compression are eliminated. The remaining protruding shapes are protected by bumpers. Safe structures, together with its low weight and slow speeds, are enough to make this robot safe. It can be mounted directly on a desktop without discrete safety devices or safety measures. This robot is a fairly good example of safety achieved simply through the design of the robot $\mathrm{arm}$. The robot is used in real-life precision assembly solutions, where a human is doing the most demanding tasks while the robot performs the monotonous and simple ones. Combining the good characteristics of human and robot, higher production is achieved. [2]

Several approaches give the same result: a light robot that cannot seriously injure a human. Several years ago, Kuka Robotics presented a small robot, the KR 3 SI, which is coated with plastic foam to absorb the kinetic energy in a collision. Inside the foam there is also a proximity sensor, which detects the vicinity of hands or other body parts. When the operator's hand comes close to the robot, the machine stops immediately and continues moving as soon as the hand is moved away. The design of the tool flange also takes safety into account; the flange includes a magnetic switch that flips down if too much force is applied to the tool. [3]

A slightly different approach to safer robotics is the German Aerospace Centre DLRs' Light-Weight Robot (LWR). Compared to the simply structured Katana, the LWR is packed with technology. Their similarity is their light weight; the LWR weighs 13.5 kilograms but can handle over 15 kilograms. The DLR robot's every 
axis is equipped with force-torque sensors, which can detect collisions along the whole arm of the robot. Alongside the axle are the controlling and other electronics related to axle movement. Upper level control and the brain are in the external computer, which makes decisions based on sensory data. This robot brings to human-robot cooperation solutions extensive collision detection and, more important,

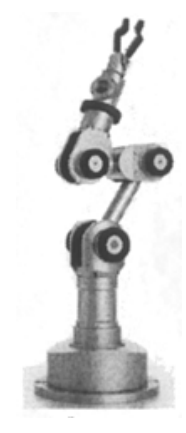

Fig. 1. Neuronics' small Katana robot [2]

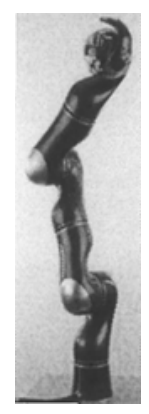

Fig. 2. DLR's LWR III [4]

options after a collision has occurred. The robot can perform a traditional emergency stop, which is not suitable in a squeeze situation. In such a case, the robot can switch to the gravity compensation mode, in which it compensates for its own weight and can be moved by simply pushing it at any point of the arm. After a collision, the robot can also change its moving direction and withdraw. A certain limit for a force that the robot directs at a collision can be specified. Due to fast servos and sensors, the collision force stays at the set value. [4]

\subsection{Software-based safety systems}

Reliable control of a robot is essential for safer robotics, especially in human-robot cooperation. To make that possible and feasible, several robot manufacturers have released robot controllers, which provide an auxiliary unit beside the normal robot controller components. The safety controller watches ceaselessly the orientation and speed of the robot and compares it to the programmed and calculated values. If there are any differences between the real-life and predicted movements of the robot, it is stopped immediately. The safety controller makes possible, for example, software-based limit switches, restricting movements in 3D space, safe reduced speeds and safe standstill in any orientation. All these new functions can be used for safety-related purposes. Even though current and even older robot controllers are able to perform some of these actions, they are not safe enough for occupational safety purposes. 


\subsection{Vision and range sensors}

The usual way to ensure the safety of a robot cell is separation. The robot is fenced in, far away from humans. The only way to get near the robot is through a door, which is strictly monitored by safety devices. This approach, however, limits the use of the robots' full potential. Human-robot cooperation is virtually impossible to achieve when distances are small and only fences are used for safeguarding. Standardisation also set strict guidelines for human and robot separation prior to EN ISO 10218-1.

The laser scanner is a commonly known safety device. It is capable of making observations in 2D fields and monitors quite a large area near the dangerous device. Two types of detection zones are warning and danger fields. The warning field warns of an approaching human, and the device is immediately stopped if movement is detected in the stopping field. A few laser scanners can in theory replace the fences and all other presence-sensing devices such as safety light curtains. The downside of the laser scanner is its limited sensing area and only 2D detection field.

A 3D detection field in safety devices has become possible since Pilz $\mathrm{GmbH}$ introduced SafetyEYETM. This sensor and detection is based entirely on machine vision. It is meant for human body detection in a freely shapeable 3D field around the supervised machine. Figure 1 clarifies how this device is used. 3D detection is done by three cameras, which observe the vicinity of the robot from above, i.e. the ceiling. The operator can freely modify the size, shape and position of detecting fields within the area of surveillance. Decisions are made by two PCs, which doublecheck the sensor data with both hard- and software. Actual decisions and signals are made by a programmable logical unit, which can send safety and normal signals depending on the detections made. This sensor is accepted in several safety classes and will be widely available soon. [5]

Machine vision will very likely be the next widely used new technology in safety devices. Research is ongoing into new technologies that could one day be used in safety devices. The Photonic Mixer Device (PMD) is a new type of sensor able to measure distances natively. The device sends a modulated light pulse, the reflections of which are measured by CMOS technology. The sensor gives distance values directly. This technology still has many limitations and problems, such as difficulty detecting a missing signal. Thus it cannot be expected to be viable as a safety device in the near future. [6] 


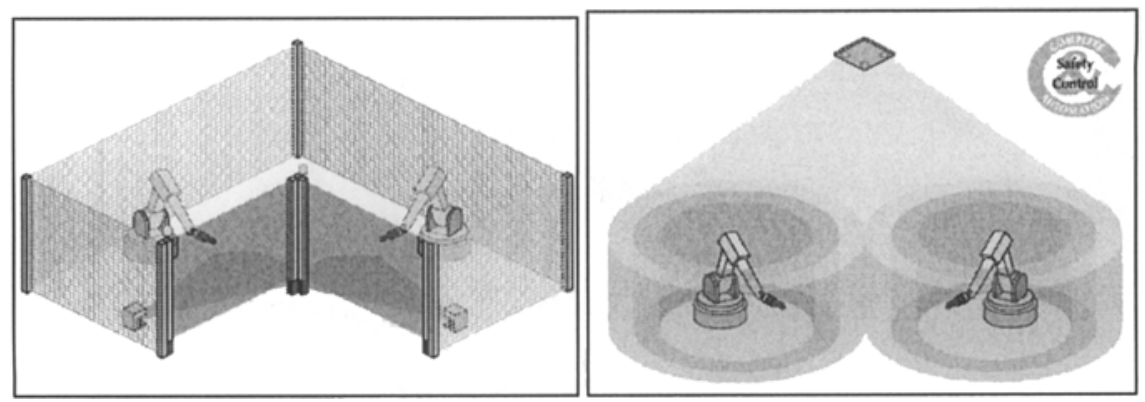

Fig. 3. Example of SafetyEYEs ${ }^{\mathrm{TM}}$ usage and advantages. [5]

\section{Development of regulations}

In Europe the Directives, and especially the Machine Directive, give the basic requirements for new machines. There are about 700 standards supporting the Directive. So-called harmonized standards interpret the requirements of the directives and set the basic level of safety. Harmonised standards do not need to be obeyed to the letter, but the level of safety should not be compromised. This may also need to be proved. Currently the most important safety standard for robots is "ISO 102181:2006. Robots for industrial environments -- Safety requirements -- Part 1: Robot". The standard was published 2006 and it gives some new aspects related to manmachine cooperation.

The safety standards aim to support development by determining physical targets for manufactures and integrators. The technical means of how to reach a physical target are open and therefore new technical solutions can appear. The requirements of new standards are quite strict, and in the near future standards will become more detailed and there will be more standards. Therefore the level of requirements will become harder since many details are already determined. This may inhibit technology development in the future, but it guarantees good and safe solutions for the industry. Detailed regulations often ease the work of the integrator, since then the safeguarding principles are well determined in standards.

Robot Standard EN ISO 10218-1 covers several features that are needed in manmachine cooperation.

Some features that the standard covers are as follows:

- Safety related parts of the control system are determined according to ISO EN 13849-1. The requirement specifies in relatively detailed level the safety features of the control system. The most common safety functions are mentioned in EN ISO 10218-1.

- Safety (reduced) speed. Basically, the speed $250 \mathrm{~mm} / \mathrm{s}$ is considered to be safe enough against impact hazard, but not against crushing, shearing or puncture hazard. Anyway, the idea of the reduced speed is that a person has enough time to avoid an impact. 
- High speed for special occasions. Special requirements for e.g. testing the robot at full speed.

- Safety distances according to EN 999 (ISO 13855). This is related to positioning the safety devices and it is possible to apply also for dynamic cases, in which the safety zone changes during operation. If the robot has a slow stopping performance the needed safety distance is also long.

- Hand guiding and related safety requirements are determined.

- The safety can be based on small forces against persons. The planar part of the robot may not initiate greater force than $150 \mathrm{~N}$ (static) or $80 \mathrm{~W}$ (impact) power against a person. The force is not much and the values can be reached only with relatively light robots and low speed. The stated values give guidelines that robot manufacturers can aim for.

- Soft limits are described. The robot can be safely under control with a programmable system (according to ISO 13849-1). This is a very important factor for safety controllers. Safety controllers make it more convenient, or in some cases make it possible, to change (safely) safety features during the operation. This can greatly help the close cooperation between a human and a robot. Such dynamic safety features must be designed into the system so that the operator cannot change it.

The standard does not cover e.g. equipment besides the robot arm or robots for other than typical industrial use. Considering man-robot cooperation, some requirements are quite hard for current robots (e.g. maximum impact energy against a person and realisation of soft limits), but the requirements set targets for robot manufacturers, and now two important safety targets are light-weight robots with quick performance in case of emergency and sophisticated safety controllers. [7]

\section{Concept solutions}

In this chapter we present five different ways to orchestrate man-robot cooperation. These are only concepts that give a general idea of the construction and in real cases many more aspects should be considered.

\subsection{Robot out of workers' reach}

One of the basic solutions to realize man-robot cooperation is to keep the robot out of workers' reach. This is the traditional way and basically it is the same idea as in the automation line, where there are manual workstations in between. The worker does his or her task and passes the product on to an automated machine or robot.

There has to be a courier between the robot and the worker. This can be a circular table as in Figure 4, or a conveyor with or without a palette. If a conveyor without a palette is used, some kind of mechanical positioning system or machine vision system must be used. In the simplest form the courier system can be a gravity-based 
mechanical slide. If the product needs to return from the robot, a returning system is needed which can also be based on any of the above solutions.

This way both the robot and the worker can carry out their tasks without interference. If there is a divergence in cycle times between the robot and the worker, the courier system can act as a buffer in unison. The downside of this solution is still the distance to the robot. Real cooperation is impossible and the structures may be very expensive.

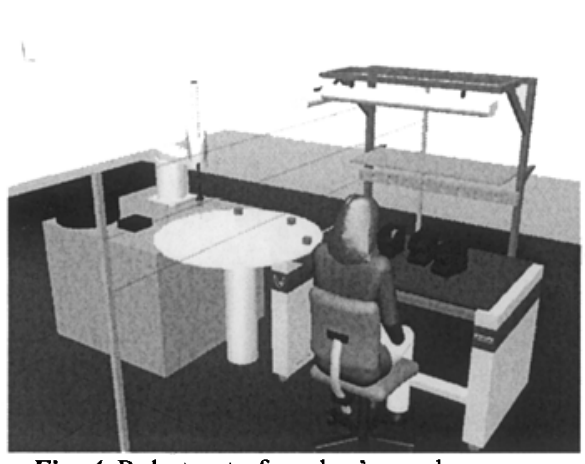

Fig. 4. Robot out of worker's reach.

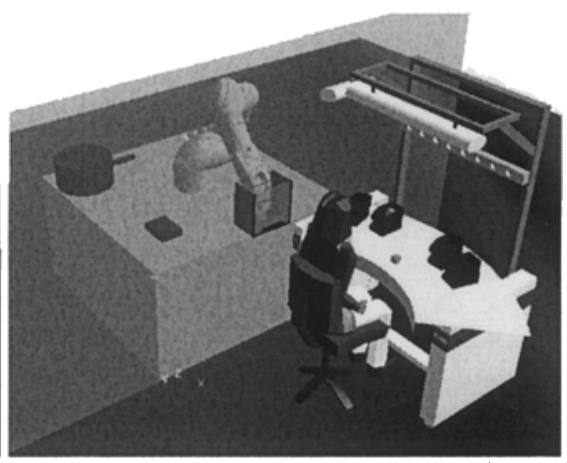

Fig. 5. Robot isolated from the worker.

\subsection{Access to robot area via a safety-controlled hatch}

If there is a plexiglass or other isolation that the robot cannot penetrate between itself and the worker, the robot can be brought closer to the worker. There should be a hatch in the partition, which both the robot and the worker can access so that exchange of material can take place. (Figure 5)

The hatch has to be such that only either the robot or the worker can access the handled material at any one time. This can be arranged mechanically so that only one side of hatchway opens at a time. The other way to do this without complex structures is to use a safety controller and a small simple door. The door is fitted with a safety sensor that sends a signal to the robot if the door is open. When the door is open, a pre-programmed area at least $500 \mathrm{~mm}$ from the worker's reach is blocked from the robot by the safety controller. If the robot happens to be in the restricted area when the door is opened, the robot is stopped or switched to safety speed by the safety controller. If the door is large enough for a person to enter, there should be a safety sensor, e.g. optical sensors active when the door is open, and when the sensors detect something the robot should be stopped.

This solution allows a person to work within very close range of the robot, and the latter solution allows humans do this without any massive safety structures. 


\subsection{Small and slow robot}

There are very light and small robots that can cooperate with people without any external safety devices. If no great force or speed is needed in a production task and a robot is justified for the task, one of these light robots can be considered. In assembly tasks this robot can act as a third hand, material handler or precision task executor. (Figure 6)

Even if there is no need for man-robot cooperation, a light robot can save costs because there is no need to isolate it from people.

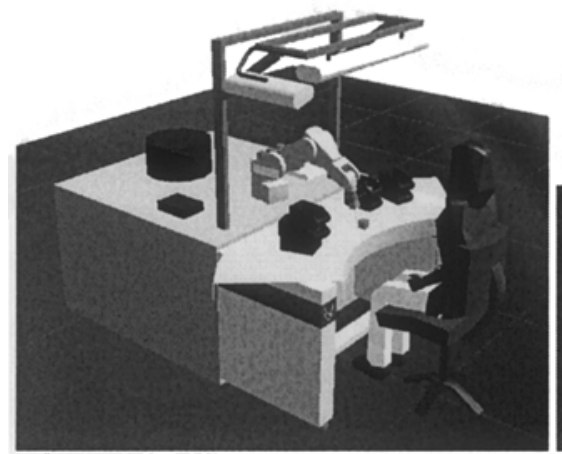

Fig. 6. Safe robot in cooperation with the worker

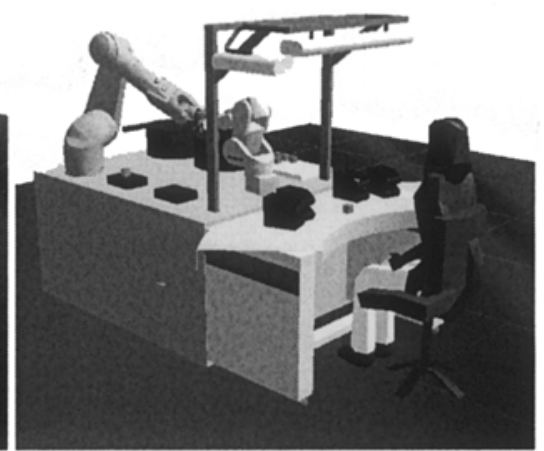

Fig. 7. Safe robot as a courier to a faster robot

\subsection{Small and slow robot as a courier}

If there is a need for man-robot cooperation and the speed of the safe and light robot is not enough, the light robot can act as a courier to a faster and stronger robot as shown in Figure 7. Even if the courier robot's only work is to act as a material transporter, often it is justified because the costs of a conveyor and its structures can climb pretty high.

In cases where one high-speed robot cannot keep up with the worker's cycle time, adding another (courier) robot that can also do production tasks may be one effective solution. Also when there is a dangerous machine these safe robots act as couriers between the worker and the machine.

\subsection{Switching safety areas}

If a robot's exact position and the surrounding workers' positions can be detected, it is possible to make a fenceless robot station where workers can work within the robot's reach. The robot's position is easy to obtain from the robot controller, and with a safety controller the position data is safety-classified. 
A human's position's acquisition is more complicated. A safety system based on ceiling-mounted vision is in many ways good for monitoring a human's position, but there are still a few drawbacks. The response time in today's vision-based safety systems is around $300 \mathrm{~ms}$, which gives quite long safety distances. But if a safety laser scanner is used which has a response time around $60 \mathrm{~ms}$, safety distances are reasonable to about $500 \mathrm{~mm}$ depending on the robot's speed and stopping distance. Also safety pressure mats are very effective in defining a worker's position.

In a safety laser scanner there are warning and stopping areas that can be predefined and changed on-the-run. Safety laser scanners do not give the position of a detected object; consequently it may be necessary to have as many scanners as working areas. When a worker approaches a workstation, entering the warning zone of a laser scanner, the safety scanner sends a signal to the robot's safety controller. If the robot is not working at that workstation, the worker is signalled that he or she is allowed to enter the station, that the station's safety scanner's stopping area is diminished, and that area is removed from the robot's work area by the safety controller. If the robot is working at the station that the worker is entering, he or she is signalled to stop, but if he or she ignores the warnings and enters the safety scanner's stopping area, the robot is immediately stopped. A laser scanner-based safety area switching system is illustrated in Figure 8. In the presented solution, it should be taken into account that the worker does not climb on to the table.

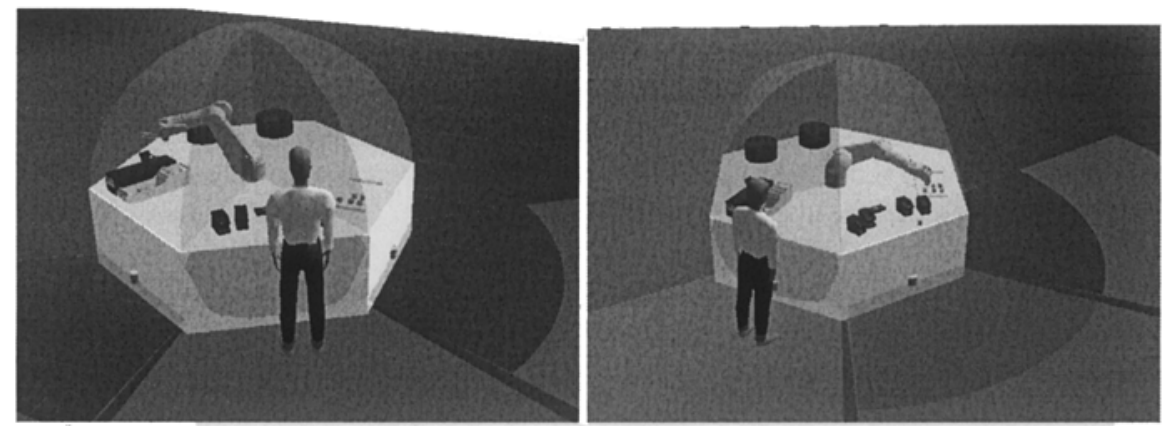

Fig. 8. A laser scanner detects the worker and switches the robot's prohibited areas accordingly.

\section{Discussion and conclusions}

Ideally, from a safety point of view, robots would be aware of surrounding people's positions and would control their own movements and speed accordingly. In that case no extra fences would be needed and robots could work in a very limited space. However, since getting a worker's exact position in real time in safetyclassified ways is not yet a reality, some compromises must be made.

By allowing software-based safety systems into the robot standard, designing these cooperative systems has become possible. Before safety controllers, the whole area around the robot was restricted from humans if the robot was not moving at safety speed. Now, safety controllers solve one problem, which is the robot's posi- 
tion and movements. If safety regulations keep on evolving, safety controllers will be able to create dynamic safety areas that surround the moving parts of the robot.

In addition to knowing the robot's position, the position of the surrounding people has to be known. The most promising solutions are vision-based safety systems. Different systems are being developed but the Piltz system is the only one on the market, and it still lacks several important features needed in man-robot cooperation. For example, the robot's working area has to be muted out from SafetyEYETM because it cannot yet distinguish a robot from a human.

Even with today's technology it is still a challenge to build a seamless flexible man-robot cooperative unit. The costs of safety system constructions may become very high and other solutions might be more cost-effective. Nevertheless, cooperative tasks like teaching or controlling the robot by moving it by hand and working within the robot's reach are becoming more common. Also the worker's perspective has to be taken into account. Even though the working area of the worker would be completely safe within the robot's reach, without fences the psychological effect that a high-speed robot creates could at the very least lower work efficiency.

Safety should be kept in mind when programming a robot. Even though a human is not working within the robot's area, the robot's movements should be somewhat predictable. Also movements in extreme positions carry much more kinetic energy, i.e. stopping distances are greater and damage on collision is more severe.

\section{References}

[1] Nof, Shimon Y. (editor) (1999). Handbook of Industrial Robotics, 2nd ed. John Wiley \& Sons. 1378 pp. ISBN 0-471-17783-0.

[2] Neuronics AG, "Neuronics AG - Katana" Internet site, available: http://www.neuronics.ch/cms_en/web/index.php?id=244\&s=katana

[3] Peter Heiligensetzer, Sichere Mensch-Roboter-Kooperation, 33. Sitzung des VDI/VDEGMAFachausschuß "Steuerung und Regelung von Robotern", 2003

[4] German Aerospace Center (DLR), "DLR - Institute of Robotics and Mechatronics . Light Weight Robots", Internet site, available: http://www.dlr.de/rmneu/en/desktopdefault.aspx/tabid-3803/

[5] Mensch und Automation - Die Zeitung für Kunden der Pilz GmbH \& Co. KG - Ausgabe 4/2006, Pilz GmbH\&Co. KG, 2006, Pp 1 - 2

[6] T. Möller, H. Kraft, J. Frey, M. Albrecht and R. Lange, Robust 3D Measurement with PMD Sensors, in Proceedings of the 1st Range Imaging Research Day, 2005.

[7] ISO 10218-1:2006. « Robots for industrial environments -- Safety requirements -- Part 1: Robot » The International Organization for Standardization (ISO). 27 p. 